Bull. Fac.Agric., Cairo Univ. 64:322- 331 (2013)

\title{
EFFECT OF SOME HERBICIDES ON ANNUAL GRASS AND BROAD LEAVED WEEDS IN MAIZE CROP I- Effect of foramsulfuron herbicide on annual grass and broad leaved weeds
}

(Received: 30.9.2013)

\author{
By \\ A.S. Marzouk \\ Phytotoxicity Department, Central Agricultural Pesticides Laboratory, \\ Agricultural Research Center, Giza, Egypt
}

\begin{abstract}
Two trials were conducted during 2010 and 2011 summer seasons in Tahanoub area, Qualubia Governorate, Egypt, to evaluate the control efficiency of different doses of the new selective herbicide Equip 22.5\% OD (foramsulfuron + isoxadifen-ethyl) against annual grass and broad leaved weeds in maize (Zea mays L.) fields compared to the recommended herbicide by the Egyptian Ministry of Agriculture; Starane $20 \%$ EC (fluroxypyr) and manual hoeing.

The results of the average density existed broad leaved weeds (BLW) and grass leaved weeds (GLW) control percentage of the $1^{\text {st }}$ and the $2^{\text {nd }}$ seasons indicated that manual hoeing treatment showed the highest control effect $(88.36 \%$ and $85.7 \%)$, followed by Equip $22.5 \%$ OD at the double recommended rate $(1500$ cc/fed.), showing 75.16 and $77.7 \%$, while when applied at the recommended rate $(750 \mathrm{cc} / \mathrm{fed}$.), it showed 71.46 and $62.1 \%$. Starane $20 \%$ EC recorded 58.1 and 45.2\%, compared to the untreated control, respectively.

Average of $\mathrm{BLW}_{\mathrm{s}}$ dry weight of the two seasons indicated that hoeing showed high reduction of BLW $\mathrm{s}_{\mathrm{s}}$ dry weight, being $92.57 \%$ of the control followed by Equip $22.5 \%$ OD at the double dose $(83.54 \%)$. and, when used at the recommended dose, showed $76.51 \%$. The minimum average reduction was obtained by Starane $20 \%$ EC reaching $59.78 \%$ of the control. Also, $\mathrm{GLW}_{\mathrm{s}}$ average dry weight was significantly reduced by hoeing $(89.9 \%)$, followed by Equip $22.5 \%$ OD at the double rate $(87.7 \%)$, while at the recommended rate it was $59.9 \%$. Starane $20 \%$ EC showed the minimum reduction percentage $(51 \%)$.
\end{abstract}

Key words: Equip 22.5\% OD, foramsulfuron + isoxadifen-ethyl, Maize plant (Zea mays L.), Starane 20\% EC, weed control, weed density.

\section{INTRODUCTION}

Maize (Zea mays L.) is the world's third most important cereal grain after wheat and rice. Maize is grown primarily for grain and secondarily for fodder. It has high nutritive value as it contains $72 \%$ starch, $10 \%$ protein, $4.8 \%$ oil, $9.5 \%$ fiber, $3 \%$ sugar and $1.7 \%$ ash (Chaudhary, 1983). Among various factors responsive for low yield, weed infestation is of supreme importance.

Maize is sensitive to weeds, especially in early growth stages (Baghestani et al., 2007). Weeds compete vigorously with maize for many resources. They reduce crop yield by competing for light, water, nutrients and carbon dioxide, interfere with harvesting and increase the cost involved in crop production. When weed populations are left uncontrolled they can substantially reduce maize yields (Hall et al., 1992 Knezevic et al., 1994, Evans et al., 2001, Halford et al., 2001). Researchers have shown that season-long interference from weeds can reduce yields by more than 30\% (Arnold, 2003).

Control of weeds in maize field is, therefore, very essential for obtaining good crop.

Weed control practices in maize resulted in 77 to $96.7 \%$ higher yield than weed check (Khan et al., 1998). Weeds can be controlled by cultural, biological and chemical measures. No doubt cultural methods are still useful tools but are laborious, time consuming and getting expensive. Moreover, the labor problem is becoming acute day by day and it will not be possible and economic to stick to the 
traditional cultural weed control practices (Oreck and Dehne, 2004; Oerke, 2005).

Keeping in view these limitations, chemical weed control is an important alternative. Herbicide application is an efficient way to check weed infestation that helps in achieving a speedy breakthrough for increasing maize production. Weed control in maize by herbicides has been suggested by many researchers (Devender et al., 1998; Toloraya et al., 2001).

A new post-emergence herbicide (Equip 22.5\% OD) was used in this study. Equip $22.5 \%$ OD is a novel sulfonylurea herbicide for post-emergence use in maize which is effective against major grass weed species, as well as some broadleaf weeds. Translocation of this herbicide takes place through leaves and roots of the weeds and then their growth stops leading to death. Post emergence herbicides are generally absorbed through leaves.

Keeping these factors in view, the present experiment is conducted to study the effect of the new post emergence herbicide Equip 22.5\% OD on weed density and growth in maize fields.

\section{MATERIAL AND METHODS}

\subsection{Field preparation and experimental design}

The trials were conducted during 2011 and 2012 summer seasons in Tahanoub area, Qualubia Governorate, Egypt, to evaluate the efficiency of the recommended rate $(750 \mathrm{cc} / \mathrm{fed}$.) and its double rate $(1500 \mathrm{cc} / \mathrm{fed}$.$) of the new herbicide foramsulfuron$ against annual grass and broad leaved weeds in maize (Zea mays L.) compared to the herbicide recommended by the Egyptian Ministry of Agriculture. All treatments were laid out in a randomized complete block design with three replications as well as the untreated check (control), having a net plot size of $54 \mathrm{~m}^{2}$. Hagen 2030 (hybrid) maize variety was used. The analysis indicated that the soil was silty clay with particle size distribution of the grown soil is $0.0 \%$ sand, $61 \%$ silt and $39 \%$ clay. Some of the physico-chemical characteristics

and organic matter content (OM) of the soil are as in Table (1).

The existed weeds were classified by the Weed Research Central Laboratory, Agricultural Research Center and divided into two groups as follows:-

\section{Group I: broad leaved weeds:}

$1^{\text {st }}$ season:

Trianthema

portulacastrum

Euphorbia geniculata.

Portulaca oleraceae.

Amaranthus spp.

\section{Group 2: grass weeds:}

\section{$1^{\text {st }}$ season: $\quad 2^{\text {nd }}$ season:}

Echinochloa colonum. Echinochloa colonum

Cyperus longus L.

Paspalum paspaloides

(Michx) Scribn.

Dactyloctenium aegyptium

(L.) P.Beauv

\subsection{Herbicide treatments}

The two herbicides were sprayed after crop and weeds emergence after 3 weeks from planting by a knapsack hand sprayer using flat fan nozzle at field capacity condition. Hoeing was done twice with the help of a hand hoe in the manual hoeing treatment when the soil was at field capacity condition after the $1^{\text {st }}$ and the $2^{\text {nd }}$ irrigation. All other agronomic practices were kept normal and uniform for all treatments.

Equip 22.5 OD was applied at the recommended rate $(750 \mathrm{cc} / \mathrm{fed}$.$) and at the double recommended$ rate $(1500 \mathrm{cc} / \mathrm{fed}$.$) , and Starane 20 \% \mathrm{EC}$ at the rate of $200 \mathrm{cc} / \mathrm{fed}$. The treated and the untreated check were replicated 3 times and distributed in a completely randomized plots.

Data regarding weed density, dry weight and control percentags were recorded from a $1 \mathrm{~m}^{2}$ area.

\subsection{Weed control efficacy}

After 21 days from application, the density of grass leaved weeds $\left(\mathrm{GLW}_{\mathrm{s}}\right)$ and broad leaved weeds

Table (1): Physico-chemical characteristics of the grown soil.

\begin{tabular}{|c|c|c|c|c|c|c|c|c|c|c|}
\hline pH & EC & \multicolumn{4}{|c|}{ Soluble cations (meq/l) } & \multicolumn{3}{|c|}{ Soluble anions (meq/l) } & \multirow{3}{*}{$\underset{\%}{\text { O.M }}$} & \multirow{3}{*}{$\begin{array}{l}\text { SP } \\
\% *\end{array}$} \\
\hline$(1: 2.5)$ & Dsm $^{-1}$ & \multirow[t]{2}{*}{$\mathrm{Ca}^{++}$} & \multirow[t]{2}{*}{$\mathbf{M g}^{++}$} & \multirow[t]{2}{*}{$\mathbf{K}^{+}$} & \multirow[t]{2}{*}{$\mathrm{Na}^{+}$} & \multirow[t]{2}{*}{$\mathrm{HCO}_{3}^{-}$} & \multirow[t]{2}{*}{$\mathrm{Cl}^{-}$} & \multirow[t]{2}{*}{$\mathrm{SO}_{4}^{--}$} & & \\
\hline Suspen & at $25 \mathrm{C}$ & & & & & & & & & \\
\hline 7.2 & 6.9 & 37.84 & 20.3 & 1.6 & 21.74 & 4.25 & 26.56 & 50.67 & 1.96 & $\mathbf{5 3 . 3 3}$ \\
\hline
\end{tabular}

* Saturation percentage 
$\left(\mathrm{BLW}_{\mathrm{s}}\right)$ were counted in each plot by using a $1 \mathrm{~m}^{2}$ frames and the average count $/ 3$ replicates of each weed was recorded. Weeds were harvested from a $1-\mathrm{m}^{-2}$ area (x3 replicates) in each plot, identified by species, then counted, and then oven dried at $72{ }^{\circ} \mathrm{C}$ for $48 \mathrm{~h}$.

Based on weed density $/ \mathrm{m}^{2}$ and dry weight $\left(\mathrm{g} / \mathrm{m}^{2}\right)$, control efficacy $\%$ of the different treatments was recorded compared to the untreated check after 3 weeks from application against each one of the existed weeds according to Henderson and Tilton (1955) equation as follows:

Control efficacy $\%=$

weed No. in cont. - weed No. in treatment $\times 100$

Weed No. in control

2.4. Statistical analysis: Data collected were analyzed statistically using SPSS analysis of variance and least significant difference test was applied at 5\% probability level to compare treatment means.

\section{RESULTS AND DISCUSSION \\ 3.1. Annual broad leaved weed density}

At the $1^{\text {st }}$ and the $2^{\text {nd }}$ seasons, the data presented in Tables (2 and 3) showed that all the treatments significantly reduced $\mathrm{BLW}_{\mathrm{s}}$ density compared to the untreated control. The effect of the treated herbicides fluctuated significantly according to the herbicide type, dose and weed group as well as species. The maximum significant weed density of all existed $\mathrm{BLW}_{\mathrm{s}}$ was recorded in the untreated check followed by Starane $20 \%$ EC. The untreated check and Starane 20\% EC treatments showed the minimum average of weed control percentage being 0.0 and $48.7 \%$, respectively. Manual hoeing showed highly significant reduction of broad leaved weed density recording the highest weed control percentage (88.36\%), followed by Equip $22.5 \%$ OD. The results of Equip $22.5 \%$ OD treatments showed no significant differences in the number of $\mathrm{BLW}_{\mathrm{s}}$ when it was applied at the double rate or at the recommended rate showing average weeds control percentage being 81.7 and $74.95 \%$ of the untreated control, respectively.

\subsection{Annual grass leaved weeds density}

All the treatments showed significant effect on the existed GLWs compared with the untreated check during the two growing seasons.

In the $1^{\text {st }}$ season, all the treatments showed insignificant effect on E. colonum density, (Tables 4 and 5). Manual hoeing treatment recorded the highest control percentage (100\%), followed by Equip $22.5 \%$ OD at the rate of $1500 \mathrm{cc} / \mathrm{fed}$., $(81.8 \%)$ and at the rate $750 \mathrm{cc} / \mathrm{fed},(54.5 \%)$, while Starane $20 \%$ EC showed the lowest control effect (45.5\%) compared to the control.

During the $2^{\text {nd }}$ season, data presented in Tables (4 and 5) showed that hoeing treatment reduced $D$. aegyptium and E. colonum density $/ \mathrm{m}^{2}$ significantly comparing with the other treatments and recorded the highest control percentage (57.32 and $78.26 \%$, respectively). Equip $22.5 \%$ OD at the rate of $1500 \mathrm{cc} /$ fed., significantly reduced $D$. aegyptium and E. colonum density compared to $750 \mathrm{cc} / \mathrm{fed}$. treatment reaching 41.09 and $62.62 \%$ of the control, respectively, while Starane $20 \%$ EC showed the lowest significant control effect (16.23 and 25.8\%, respectively).

Density of $P$. paspaloides, was reduced significantly by Equip $22.5 \%$ OD treatment (1500 cc/ fed.) which achieved the highest control percentage (86.53). Hoeing showed insignificant effect on $P$. paspaloides, density and control percentage comparing with Equip 22.5\% OD (750 cc/fed.), being 73.06 and $73.06 \%$, respectively. The minimum significant density reduction of $P$. paspaloides, was obtained by Starane $20 \%$ EC treatment record $29.14 \%$ of the control.

From the data of the average $\mathrm{BLW}_{\mathrm{s}}$ and $\mathrm{GLW}_{\mathrm{s}}$ control percentages during the $1^{s t}$ and the $2^{\text {nd }}$ seasons, it was concluded that the manual hoeing treatment showed the highest control effect $(88.36$ and $85.7 \%$ ), followed by Equip $22.5 \%$ OD at the double rate $(75.16$ and $77.7 \%)$, and the recommended rate (71.46 and $62.1 \%$ ), respectively. Starane 20\% EC showed the least effect being 58.1 and $45.2 \%$, respectively.

\section{3. Annual broad leaved weed dry weight}

The data presented in Tables (6 and7) revealed that all $\mathrm{BLW}_{\mathrm{s}}$ weeds showed the same pattern against all treatments. Hoeing and Equip 22.5 OD significantly reduced the total dry weight $/ \mathrm{m}^{2}$ of the broad leaved weeds after 21 days from application compared with Staranee 20\% EC and the control treatments. Also, Equip 22.5\% OD at the double rate was significantly effective than the low rate. Hoeing treatment showed the least significant effect on dry weight being $15.26 \mathrm{~g}$ and $0.5 \mathrm{~g} / \mathrm{m}^{2}$, while Starane $20 \%$ EC achieved the highest effect on dry weight reaching 74.29 and $5.4 \mathrm{~g} / \mathrm{m}^{2}$ at the $1^{\text {st }}$ and the $2^{\text {nd }}$ season, respectively. 
Table ( 2 ) : Average denisty of broad leaved weeds in maize field as influnced by herbicide treatments after 21 days from application.

\begin{tabular}{|c|c|c|c|c|c|c|c|c|c|c|}
\hline \multicolumn{2}{|c|}{ Treatment } & \multicolumn{9}{|c|}{ Weed No./ $\mathbf{m}^{2}$} \\
\hline \multirow[b]{2}{*}{ Herbicide } & \multirow{2}{*}{$\begin{array}{c}\text { Rate of Appl. } \\
\text { CC /fed. }\end{array}$} & \multicolumn{5}{|c|}{$\mathbf{1}^{\text {st }}$ Season } & \multicolumn{4}{|c|}{$2^{\text {nd }}$ Season } \\
\hline & & $\begin{array}{c}T . \\
\text { ptul. }\end{array}$ & $\begin{array}{c}P . \\
\text { oler. }\end{array}$ & E. gen. & $\begin{array}{l}A . \\
\text { Spp }\end{array}$ & $\begin{array}{c}D . \\
\text { stra. }\end{array}$ & $\begin{array}{l}\text { C. } \\
\text { olit. }\end{array}$ & E. gen. & T. ptul. & $\begin{array}{c}\text { C. } \\
\text { arve. }\end{array}$ \\
\hline Equip22.5 OD & 750 & 51.90 c & $1.15 \mathrm{c}$ & $2.3 \mathrm{c}$ & $2.10 \mathrm{c}$ & $2.25 \mathrm{c}$ & 0.00 c & 0.34 b & 0.0 & $1.42 \mathrm{~b}$ \\
\hline Equip 22.5 OD & 1500 & $47.15 \mathrm{c}$ & $1.00 \mathrm{c}$ & $1.9 \mathrm{c}$ & $1.90 \mathrm{c}$ & $0.92 \mathrm{~d}$ & 0.00 c & 0.00 b & 0.0 & $1.75 \mathrm{~b}$ \\
\hline Starane $20 \%$ EC & 200 & $103.8 \mathrm{~b}$ & 3.37 b & $4.1 \mathrm{~b}$ & $4.30 \mathrm{~b}$ & $3.84 \mathrm{~b}$ & $0.42 \mathrm{~b}$ & $0.42 \mathrm{~b}$ & 0.0 & $2.59 \mathrm{a}$ \\
\hline Manual hoeing & Twice & $17.90 \mathrm{c}$ & $0.30 \mathrm{c}$ & 0.9 c & $1.10 \mathrm{~d}$ & $0.33 \mathrm{~d}$ & 0.42 b & 0.00 b & 0.0 & $0.42 \mathrm{c}$ \\
\hline Control & -------- & $202.75 \mathrm{a}$ & $5.25 \mathrm{a}$ & $7.5 \mathrm{a}$ & $7.75 \mathrm{a}$ & $7.25 \mathrm{a}$ & 0.84 a & $0.84 \mathrm{a}$ & 0.0 & $2.92 \mathrm{a}$ \\
\hline LSD & & 41.784 & 1.612 & 1.519 & 0.679 & 0.706 & 0.332 & 0.404 & 0.000 & 0.823 \\
\hline
\end{tabular}

The figures followed by the same letters are insignificant.

Table (3): Broad weed control \% based on weed density in maize treated plots after 21 days from application.

\begin{tabular}{|c|c|c|c|c|c|c|c|c|c|c|c|}
\hline \multicolumn{2}{|c|}{ Treatment } & \multicolumn{9}{|c|}{ Weed control \% } & \multirow{3}{*}{$\begin{array}{l}\text { Average } \\
\text { weeds } \\
\text { control \% }\end{array}$} \\
\hline \multirow[b]{2}{*}{ Herbicide } & \multirow{2}{*}{$\begin{array}{l}\text { Rate of } \\
\text { Appl. } \\
\text { CC /fed. }\end{array}$} & \multicolumn{5}{|c|}{$\mathbf{1}^{\text {st }}$ Season } & \multicolumn{4}{|c|}{$2^{n d}$ Season } & \\
\hline & & $\begin{array}{l}\text { T. } \\
\text { ptul. }\end{array}$ & $\begin{array}{l}P . \\
\text { oler. }\end{array}$ & $\begin{array}{l}\text { E. } \\
\text { gen. }\end{array}$ & $\begin{array}{l}A . \\
\text { Spp }\end{array}$ & $\begin{array}{l}\text { D. } \\
\text { stra. }\end{array}$ & $\begin{array}{l}\text { C. } \\
\text { olit. }\end{array}$ & $\begin{array}{l}\text { E. } \\
\text { gen. }\end{array}$ & $\begin{array}{l}T . \\
\text { ptul. }\end{array}$ & $\begin{array}{l}\text { C. } \\
\text { arve. }\end{array}$ & \\
\hline $\begin{array}{l}\text { Equip22.5 } \\
\text { OD }\end{array}$ & 750 & 74.4 & 78.1 & 69.3 & 72.9 & 69.0 & $\begin{array}{l}100 . \\
0\end{array}$ & 59.5 & 100.0 & 51.4 & 74.95 \\
\hline $\begin{array}{l}\text { Equip } 22.5 \\
\text { OD }\end{array}$ & 1500 & 76.7 & 81 & 74.7 & 75.5 & 87.3 & $\begin{array}{l}100 . \\
0\end{array}$ & 100.0 & 100.0 & 40.1 & 81.70 \\
\hline $\begin{array}{l}\text { Starane } 20 \% \\
\text { EC }\end{array}$ & 200 & 48.8 & 35.7 & 45.3 & 44.5 & 47.0 & 50.0 & 50.0 & 100.0 & 11.3 & 48.07 \\
\hline $\begin{array}{l}\text { Manual } \\
\text { hoeing }\end{array}$ & Twice & 96.1 & 94.3 & 88 & 85.8 & 95.4 & 50.0 & 100.0 & 100.0 & 85.6 & 88.36 \\
\hline Control & -------- & 0.0 & 0.0 & 0.0 & 0.0 & 0.0 & 0.0 & $\mathbf{0 . 0}$ & 0.0 & 0.0 & 0.00 \\
\hline
\end{tabular}


Table (4): Average denisty of grass leaved weeds in maize field as influnced by herbicide treatments after 21 days from application.

\begin{tabular}{|c|c|c|c|c|c|c|}
\hline \multirow{2}{*}{\multicolumn{2}{|c|}{ Treatment }} & \multicolumn{5}{|c|}{ Weed No./ m2 } \\
\hline & & \multirow{2}{*}{$\begin{array}{l}\mathbf{1}^{\text {st }} \text { Season } \\
\text { E. colo. }\end{array}$} & \multicolumn{4}{|c|}{$2^{\text {nd }}$ Season } \\
\hline Herbicide & $\begin{array}{c}\text { Rate of Appl. } \\
C C / f e d .\end{array}$ & & D. aeg. & E. colo. & P. pasp. & C. long. \\
\hline Equip22.5 OD & 750 & $1.25 \mathrm{a}$ & $4.25 \mathrm{~b}$ & $40.58 \mathrm{c}$ & $1.84 \mathrm{c}$ & 0.0 \\
\hline Equip 22.5 OD & 1500 & $0.50 \mathrm{a}$ & $3.34 \mathrm{c}$ & $28.50 \mathrm{~d}$ & $0.92 \mathrm{~d}$ & 0.0 \\
\hline $\begin{array}{c}\text { Starane } 20 \% \\
\text { EC }\end{array}$ & 200 & $1.50 \mathrm{a}$ & $4.75 \mathrm{~b}$ & $56.58 \mathrm{~b}$ & $4.84 \mathrm{~b}$ & 0.0 \\
\hline Manual hoeing & Twice & 0.00 a & $2.42 \mathrm{~d}$ & $16.58 \mathrm{e}$ & $1.84 \mathrm{c}$ & 0.0 \\
\hline Control & ----- & $2.75 \mathrm{a}$ & $5.67 \mathrm{a}$ & $76.25 \mathrm{a}$ & $6.83 \mathrm{a}$ & 0.0 \\
\hline LSD (0.05 ) & & 3.043 & 0.847 & 1.883 & 0.558 & 0.000 \\
\hline
\end{tabular}

The figures followed by the same letters are insignificant.

Table (5) : Grass weed control \% based on grass leaved weed density in maize treated plots after 12 days from application.

\begin{tabular}{|c|c|c|c|c|c|c|l|}
\hline \multicolumn{2}{|c|}{ Treatment } & \multicolumn{5}{c|}{ \% of control } & \multicolumn{2}{c|}{$\begin{array}{l}\text { Average } \\
\text { weed }\end{array}$} \\
\cline { 2 - 8 } Herbicide & $\begin{array}{c}\text { Rate of } \\
\text { Appl. } \\
\text { CC /fed. }\end{array}$ & E. colo. & D. aeg. & E. colo. & P. pasp. & C. long. & \multirow{2}{*}{ Contraol \% } \\
\hline Equip 22.5 OD & $\mathbf{7 5 0}$ & $\mathbf{5 4 . 5}$ & $\mathbf{2 5 . 0 4}$ & $\mathbf{4 6 . 7 8}$ & $\mathbf{7 3 . 0 6}$ & $\mathbf{1 0 0 . 0 0}$ & $\mathbf{6 2 . 1}$ \\
\hline Equip 22.5 OD & $\mathbf{1 5 0 0}$ & $\mathbf{8 1 . 8}$ & $\mathbf{4 1 . 0 9}$ & $\mathbf{6 2 . 6 2}$ & $\mathbf{8 6 . 5 3}$ & $\mathbf{1 0 0 . 0 0}$ & $\mathbf{7 7 . 7}$ \\
\hline $\begin{array}{c}\text { Starane 20\% } \\
\text { EC }\end{array}$ & $\mathbf{2 0 0}$ & $\mathbf{4 5 . 5}$ & $\mathbf{1 6 . 2 3}$ & $\mathbf{2 5 . 8 0}$ & $\mathbf{2 9 . 1 4}$ & $\mathbf{1 0 0 . 0 0}$ & $\mathbf{4 5 . 2}$ \\
\hline Manual hoeing & Twice & $\mathbf{1 0 0}$ & $\mathbf{5 7 . 3 2}$ & $\mathbf{7 8 . 2 6}$ & $\mathbf{7 3 . 0 6}$ & $\mathbf{1 0 0 . 0 0}$ & $\mathbf{8 5 . 7}$ \\
\hline Control & & $\mathbf{0 . 0}$ & $\mathbf{0 . 0 0}$ & $\mathbf{0 . 0 0}$ & $\mathbf{0 . 0 0}$ & $\mathbf{0 . 0 0}$ & $\mathbf{0 . 0}$ \\
\hline
\end{tabular}

The figures followed by the same letters are insignificant.

The average weed control percentages of the two seasons (Tables 6 and 7) indicated that hoeing showed the highest significant reduction of $\mathrm{BLW}_{\mathrm{s}}$ dry weight being $92.57 \%$ of the control, followed by Equip $22.5 \%$ OD at the higher rate $(83.54 \%)$ and Equip $22.5 \%$ OD at the lower rate (76. 51\%), while the minimum average reduction was obtained by Starane $20 \%$ EC reaching $59.78 \%$ of the control.

\subsection{Annual grass leaved weed dry weight}

At the $1^{\text {st }}$ season (Tables 8 and 9), all treatments showed no significant effect on GLW $_{\mathrm{s}}$ (E. colonum.). The highest reduction percentage was obtained with hoeing reaching $100 \%$ of the control, followed by Equip $22.5 \%$ OD at the double rate $(88.9 \%)$. The recommended rate of Equip $22.5 \%$
OD and Starane 20\% EC showed the same reduction percentage in E. colonum, dry weight being $33.3 \%$ of the control.

During the $2^{\text {nd }}$ season all treatments showed the same effect on D. aegyptium. and E. colonum. (Tables 8 and 9). Hoeing showed the highest significant reduction of $D$. aegyptium. and $E$. colonum dry weight reaching 86.85 and $59.33 \%$ of the control, followed by Equip 22.5\% OD at the rate of $1500 \mathrm{cc}$, being 78.90 and $56.9 \%$ of the control, respectively. Equip $22.5 \%$ OD at the double rate caused more herbicidal reduction on the dry weight against $P$. paspaloides , reaching $96.19 \%$, followed by $83.45 \%$ in manual hoeing, Starane $20 \%$ EC $(44.6 \%)$ and Equip $22.5 \%$ OD at the recommended 
Table (6): Average $\mathrm{dw}\left(\mathrm{gm} / \mathrm{m}^{2}\right)$ of broad leaved weeds in maize field as influnced by herbicide treatments after 21 days from treatment.

\begin{tabular}{|c|c|c|c|c|c|c|c|c|c|c|c|c|}
\hline \multicolumn{2}{|c|}{ Treatment } & \multicolumn{11}{|c|}{ Dry weight gm / m² } \\
\hline \multirow[b]{2}{*}{ Herbicide } & \multirow{2}{*}{$\begin{array}{c}\text { Rate of } \\
\text { Appl. } \\
\text { CC /fed. }\end{array}$} & \multicolumn{4}{|c|}{$\mathbf{1}^{\text {st }}$ Season } & \multirow{2}{*}{$\begin{array}{c}\text { Total } \\
\text { GLW } \\
\text { dw/m } \\
\end{array}$} & \multicolumn{5}{|c|}{$2^{\text {nd }}$ Season } & \multirow{2}{*}{$\begin{array}{l}\text { Total } \\
\text { GLW } \\
\text { dw/m } \\
\end{array}$} \\
\hline & & $\begin{array}{c}T . \\
\text { ptul. }\end{array}$ & $\begin{array}{c}P . \\
\text { oler. }\end{array}$ & $\begin{array}{c}E . \\
\text { gen. }\end{array}$ & $\begin{array}{c}A \\
S p p\end{array}$ & & $\begin{array}{c}\text { D. } \\
\text { stra. }\end{array}$ & $\begin{array}{c}C . \\
\text { olit. }\end{array}$ & $\begin{array}{c}\text { E. } \\
\text { gen. }\end{array}$ & $\begin{array}{c}T . \\
p t u l .\end{array}$ & $\begin{array}{c}C . \\
\text { arve. }\end{array}$ & \\
\hline Equip22.5 OD & 750 & $45.51 \mathrm{bc}$ & $0.11 \mathrm{c}$ & $0.47 \mathrm{bc}$ & $2.00 \mathrm{c}$ & 48.09 bc & $1.77 \mathrm{c}$ & $0.00 \mathrm{c}$ & 0.19 c & 0.0 & 0.39 b & $2.4 \mathrm{c}$ \\
\hline Equip 22.5 OD & 1500 & 29.84 c & 0.12 c & $0.44 \mathrm{bc}$ & $2.07 \mathrm{c}$ & $32.47 \mathrm{c}$ & $0.53 \mathrm{~d}$ & $0.00 \mathrm{c}$ & $0.00 \mathrm{~d}$ & 0.0 & $0.28 \mathrm{c}$ & $0.8 \mathrm{~d}$ \\
\hline Starane $20 \%$ EC & 200 & 70.06 b & 0.32 b & 0.74 b & $3.17 \mathrm{~b}$ & $74.29 \mathrm{~b}$ & $4.55 \mathrm{~b}$ & $0.13 \mathrm{~b}$ & $0.28 \mathrm{~b}$ & 0.0 & $0.47 \mathrm{ab}$ & $5.4 \mathrm{~b}$ \\
\hline Manual hoeing & Twice & $14.26 \mathrm{~cd}$ & $0.06 \mathrm{c}$ & 0.18 c & $0.76 \mathrm{~d}$ & $15.26 \mathrm{c}$ & $0.34 \mathrm{~d}$ & $0.14 \mathrm{~b}$ & $0.00 \mathrm{~d}$ & 0.0 & $0.06 \mathrm{~d}$ & $0.5 \mathrm{~d}$ \\
\hline Control & ------- & 188.825 a & 0.70 a & $1.68 \mathrm{a}$ & $6.90 \mathrm{a}$ & $198.11 \mathrm{a}$ & $7.84 \mathrm{a}$ & 0.97 a & 0.98 a & 0.0 & 0.53 a & $10.3 \mathrm{a}$ \\
\hline LSD & & 26.257 & 0.190 & 0.424 & 0.385 & 26.200 & 0.368 & 0.070 & 0.049 & 0.000 & 0.085 & 0.760 \\
\hline
\end{tabular}

The figures followed by the same letters are insignificant.

Table (7): Broad weed control \% based on dry weight in maize treated plots 21 days from application.

\begin{tabular}{|c|c|c|c|c|c|c|c|c|c|c|c|}
\hline \multicolumn{2}{|c|}{ Treatment } & \multicolumn{9}{|c|}{ Weed control \% } & \multirow{3}{*}{$\begin{array}{c}\text { Average } \\
\text { BLW }_{\mathrm{s}} \\
\text { control \% }\end{array}$} \\
\hline \multirow{2}{*}{ Herbicide } & \multirow{2}{*}{$\begin{array}{c}\text { Rate of } \\
\text { Appl. } \\
\text { CC /fed. }\end{array}$} & \multicolumn{5}{|c|}{$\mathbf{1}^{s t}$ Season } & \multicolumn{4}{|c|}{$2^{\text {nd }}$ Season } & \\
\hline & & T. ptul. & P. oler. & E. gen. & A. Spp & D. stra. & C. olit. & E. gen. & T. ptul. & C. arve. & \\
\hline $\begin{array}{c}\text { Equip22.5 } \\
\text { OD }\end{array}$ & 750 & 75.9 & 85.0 & 72.0 & 71.0 & 77.4 & 100.0 & 80.6 & 100.0 & 26.7 & 76.51 \\
\hline $\begin{array}{c}\text { Equip } 22.5 \\
\text { OD }\end{array}$ & 1500 & 84.2 & 82.9 & 73.8 & 70.0 & 93.3 & 100.0 & 100.0 & 100.0 & 47.6 & 83.54 \\
\hline $\begin{array}{l}\text { Starane } \\
20 \% \text { EC }\end{array}$ & 200 & 62.9 & 54.6 & 56.0 & 54.1 & 42.0 & 86.5 & 71.4 & 100.0 & 10.5 & 59.78 \\
\hline $\begin{array}{c}\text { Manual } \\
\text { hoeing }\end{array}$ & Twice & 92.4 & 92.1 & 89.3 & 89.0 & 95.7 & 86.0 & 100.0 & 100.0 & 88.6 & 92.57 \\
\hline Control & ------- & 0.0 & 0.0 & 0.0 & 0.0 & 0.0 & 0.0 & 0.0 & 0.0 & 0.0 & 0.00 \\
\hline
\end{tabular}


Table (8): Average $\mathrm{dw}\left(\mathrm{gm} / \mathrm{m}^{2}\right)$ of grass leaved weeds in maize field as influnced by herbicide treatments after 21 days from application.

\begin{tabular}{|c|c|c|c|c|c|c|c|}
\hline \multirow{2}{*}{\multicolumn{2}{|c|}{ Treatment }} & \multicolumn{6}{|c|}{ Dry weight gm / m² } \\
\hline & & \multirow{2}{*}{$\begin{array}{c}\mathbf{1}^{\text {st }} \text { Season } \\
\text { E. colo. }\end{array}$} & \multicolumn{4}{|c|}{$2^{\text {nd }}$ Season } & \multirow{2}{*}{$\begin{array}{c}\text { Total } \\
\text { GLW } \\
\text { dw/m }\end{array}$} \\
\hline Herbicide & $\begin{array}{c}\text { Rate of Appl. } \\
\text { CC /fed. }\end{array}$ & & D. aeg. & E. colo. & P. pasp. & C. long. & \\
\hline Equip22.5 OD & 750 & $0.30 \mathrm{a}$ & $2.60 \mathrm{c}$ & 10.77 c & 0.79 b & 0.0 & $14.16 \mathrm{c}$ \\
\hline Equip 22.5 OD & 1500 & 0.05 a & $1.54 \mathrm{~d}$ & $9.75 \mathrm{~cd}$ & $0.05 \mathrm{~d}$ & 0.0 & $11.34 d$ \\
\hline Starane $20 \%$ EC & 200 & $0.30 \mathrm{a}$ & $4.75 \mathrm{~b}$ & $14.64 \mathrm{~b}$ & 0.77 b & 0.0 & 20.16 b \\
\hline Manual hoeing & Twice & 0.0 a & $0.96 \mathrm{e}$ & $9.20 \mathrm{~d}$ & $0.25 \mathrm{c}$ & 0.0 & $10.41 \mathrm{~d}$ \\
\hline Control & & 0.45 a & $7.30 \mathrm{a}$ & $22.62 \mathrm{a}$ & $1.39 \mathrm{a}$ & 0.0 & $31.31 \mathrm{a}$ \\
\hline LSD (0.05 ) & & 0.483 & 0.558 & 0.926 & 0.190 & 0.000 & 2.352 \\
\hline
\end{tabular}

Table (9): Grass weed control \% based on dry weight in maize treated plots 21 days from application.

\begin{tabular}{|c|c|c|c|c|c|c|c|}
\hline \multirow{2}{*}{\multicolumn{2}{|c|}{ Treatment }} & \multicolumn{5}{|c|}{ Weed control \% } & \multirow{3}{*}{$\begin{array}{c}\text { Average } \\
\text { GLW }_{\text {s }} \\
\text { control\% }\end{array}$} \\
\hline & & \multirow{2}{*}{$\begin{array}{c}\mathbf{1}^{\text {st }} \text { Season } \\
\text { E. colo. }\end{array}$} & \multicolumn{4}{|c|}{$2^{n d}$ Season } & \\
\hline Herbicide & $\begin{array}{c}\text { Rate of Appl. } \\
\text { CC /fed. }\end{array}$ & & D. aeg. & E. colo. & P. pasp. & C. long. & \\
\hline Equip22.5 OD & 750 & 33.3 & 64.38 & 52.39 & 42.81 & 100.00 & 59.9 \\
\hline Equip 22.5 OD & 1500 & 88.9 & 78.90 & 56.90 & 96.19 & 100.00 & 87.7 \\
\hline Starane $20 \%$ EC & 200 & 33.3 & 34.93 & 35.28 & 44.60 & 100.00 & 51.0 \\
\hline Manual hoeing & Twice & 100 & 86.85 & 59.33 & 83.45 & 100.00 & 89.9 \\
\hline Control & & 0.0 & 0.00 & 0.00 & 0.00 & 0.00 & 0.0 \\
\hline
\end{tabular}


rate being $42.81 \%$ of the untreated control.

Data in Table (9) illustrated that the maximum reduction in $\mathrm{GLW}_{\mathrm{s}}$ was obtained by hoeing (89.9\%), followed by Equip $22.5 \%$ OD at the double rate $(87.7 \%)$, Equip $22.5 \%$ at the recommended rate $(59.9 \%)$ and Starane $20 \%$ EC (51\%) of the untreated control.

The above mentioned results agree with Arnold el al. (2005), who mentioned that the controlling effect of herbicide treatments (nicosulfuron plus rimsulfuron, DPX 79406, and foramsulfuron) differed according to the herbicide treated and weed kinds. This variability in weed response (tolerance to herbicides) could be partially associated with growth rate differences among the specie (Damalas et al., 2008), which may be responsible for differences in herbicide metabolism rate. Weed tolerance to herbicides is often associated with metabolic processes that result in herbicide degradation by the target plants (Devine et al., 1993) and thus weed species can exhibit different levels of tolerance to a given herbicide even if they are similarly susceptible at their target site.

Also, Damalas et al. (2010), concluded that satisfactory control of early watergrass and late watergrass in corn can be achieved with increased application rates of foramsulfuron applied preferably at early growth stage, while McCullough et al. (2012) showed that foramsulfuron applications controlled goosegrass $<55 \%$.

The results of both two seasons showed that herbicide application caused significant effect on weed dry weight. In two seasons, weed biomass obtained was significantly different from one treatment to another. These results are in agreement with Zaremohazabieh and Ghadiri, (2011), who indicated that foramsulfuron at $0.06 \mathrm{~kg}$ a.i. $/ \mathrm{ha}^{-1}$, foramsulfuron at $0.03 \mathrm{~kg}$ a.i. / $\mathrm{ha}^{-1}$, and atrazine plus alachlor at both applied rates provided better weed control compared with the other treatments. Also, Baghestani et al. (2007) indicated that nicosulfuron and foramsulfuron at 80 and $562.5 \mathrm{~g}$ a.i. $\mathrm{P} \mathrm{ha}^{-1}$, respectively, provided satisfactory control of broadleaved and grass weeds and rimsulfuron at 10 $\mathrm{g}$ a.i. $\mathrm{P} \mathrm{ha}{ }^{-1}$ controlled grass weeds in maize. Zaremohazabieh and Ghadiri (2011), indicated that the maximum weed biomass reduction was obtained with foramsulfuron in both applied rates $(0.03$ and $0.06 \mathrm{~kg}$ a.i. $\left.\mathrm{P} \mathrm{ha}^{-1}\right)$. Also, the effectiveness of overall weed control was primarily due to lower weed population densities and a shorter duration of weed emergence after planting and therefore less reinfestation after herbicide application.

Lotfi et al. (2010), indicated that the most effective herbicide for weed density and weed dry weight decreasing percentage was Foramsulfuron which was significantly different from other herbicides. Also, factors including herbicide, application doses and cultivation significantly influenced weed density and dry weight. The best herbicide reduced weed density and decreased dry weight percentage was Foramsulfuron, and the best effective dose was $25 \%$ more than the recommended dose (Lotfi et al., 2012).

From the above mentioned results it could be concluded that satisfactory control of $\mathrm{BLW}_{\mathrm{s}}$ and $\mathrm{GLW}_{\mathrm{s}}$ in maize can be achieved with increased application rates of foramsulfuron applied preferably at early growth stage but the side effect on maize has to be considered.

\section{REFERENCES}

Arnold R. N. (2003). Broadleaf weed control in field corn with postemergence herbicides. Pages 15-18 in: 2003 Annual Weeds Research Report. Agric. Sci. Center at Farmington, Univ. of New Mex.

Arnold R. N., Smeal D. and O'Neill M. K. (2005). Broadleaf weed control in field corn with postemergence herbicides. Online. Crop Management doi:10.1094/CM-2005-0224-01RS.

Baghestani M. A., Zand E., Soufizadeh S., Eskandari A., PourAzar R., Veysi M. and Nassirzadeh N. (2007). Efficacy evaluation of some dual purpose herbicides to control weeds in maize (Zea mays L.). Crop Protection. 26: 936-942.

Chaudhary A.R. (1983). Maize in Pakistan. Punjab Agri. Coordination Board, University of Agriculture, Faisalabad.

Damalas C.A., Dhima K.V. and Eleftherohorinos I.G. (2008). Morphological and physiological variation among species of the genus Echinochloa in northern Greece. Weed Sci. 56: 416-423.

Damalas C. A., Lithourgidis A. S. and Lithourgidis C. S. (2010). Early watergrass (Echinochloa oryzoides) and late watergrass (Echinochloa phyllopogon) control with foramsulfuron. Pak. J. Weed Sci. Res. 16 (2): 161-168.

Devender S., Tyagi R.C., Agarwal S. K. and Singh 
D. (1998). Weed control methods in spring maize. Haryana Agric. Uni. J. Res. 28(1): 2125.

Devine M., Duke S.O. and Fedtke C. (1993). Physiology of herbicide action. Prentice Hall, Englewood Cliffs, NJ, pp. 441.

Evans J. O., Hauderlie P. and Williams R. W. (2001). Control of common lambsquarters in Roundup ready corn. W. Soc. Weed Sci. 2001 Res. Prog. Rep.

Halford C., Hamill A. S., Zhang J. and Doucet C. (2001). Critical period of weed control in notill soybean (Glycine max) and corn (Zea mays). Weed Technol. 15:737-744.

Hall M. R., Swanson C. J. and Anderson G. W. (1992). The critical period of weed control in grain corn (Zea mays). Weed Sci. 40:441-447.

Henderson G. F. and Tilton E. W. (1955). Test with acaricides against the brown wheat mite. J. Econ. Ent. 48: 157-160.

Khan S.A., Hussain N., Khan I.A., Khan M. and Iqbal M. (1998). Study on weed control in maize. Sarhad J. Agri. 14(6): 581-586.

Knezevic S. Z., Weise S. F. and Swanson C. J. (1994). Interference of redroot pigweed (Amaranthus retroflexus) in Corn (Zea mays). Weed Sci. 42:568-573.

Lotfi M., Daneshian J. and Baghestani M. (2012). Investigation of integrated weed management in Broomcorn ( Sorghum bicolor) farms in
Miyaneh region. J. of Agri. Sci. (univ. of Tabriz). 22(1): 55-70.

Lotfi M., Shayestenia A., Danehian J. and Moradi A. A. (2010). Effect of three post-emergence herbicides and cultivation on weed management in silage corn fields. J. of New Agri. Sci, (Modern Science of Sustainable Agriculture). 6(19): 71-77.

McCullough P. E., de Barreda D. G. and Raymer P. (2012). Nicosulfuron Use with Foramsulfuron and Sulfentrazone for late summer goosegrass (Eleusine indica) control in bermudagrass and seashore paspalum. Weed Technology: AprilJune. 26 (2): 376-381.

Oerke E.C. (2005). Crop losses to pest. J. Agri. Sci. 143: 1-13.

Oerke E.C. and Dehne H.W. (2004). Safeguarding Production- losses in major crops and the role of crop production. Crop Protec. 23: 275-285.

Toloraya T.R., Malakanova V.P. and Akhtyrstev M.G. (2001). Effectiveness of dates, methods and doses of applying Zinc sulphate and its combination with the selective herbicides Titus in maize sowings. Kukuruza-I-Sorg. 2: 5-7.

Zaremohazabieh S. and Ghadiri H. (2011). Effects of Rimsulfuron, Foramsulfuron and conventional herbicides on weed control and maize yield at three planting dates. J. Biol. Environ. Sci. 5(14): 47-56.

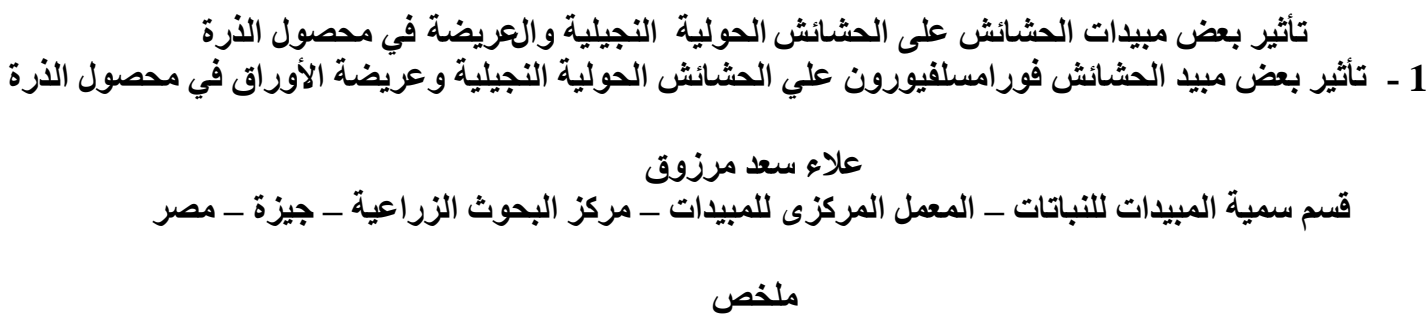

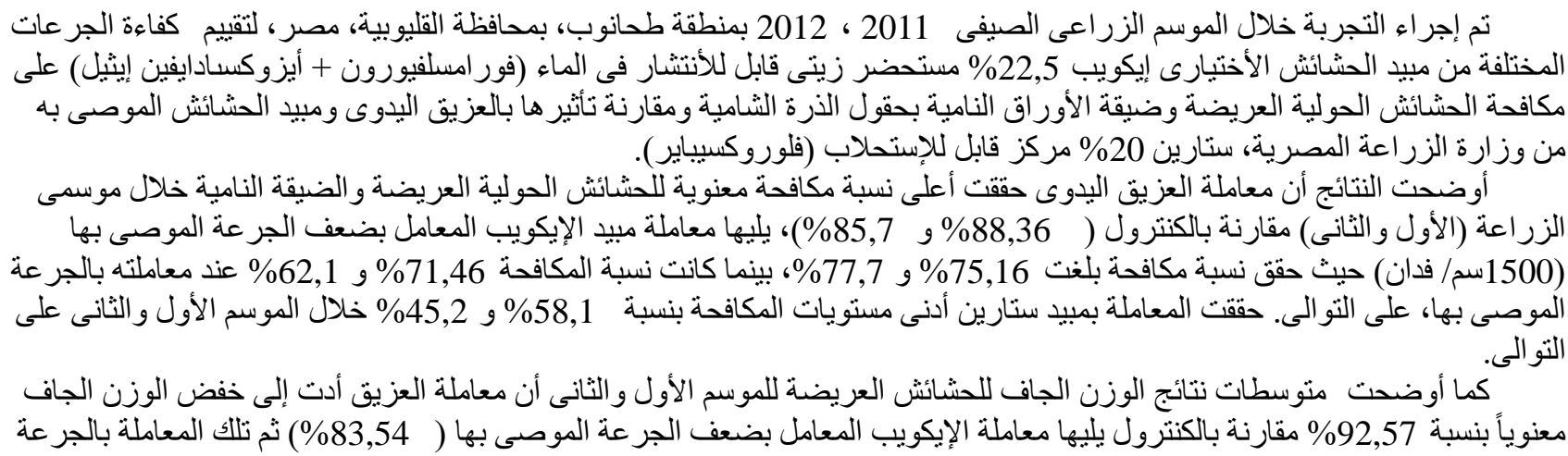




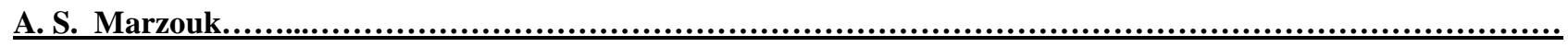

الموصى بها ( 16,51\%) و أخير اً مبيد ستارين الذى كان أقل تأثير اً على الوزن الجاف حيث أدى إلى خفض الوزن الجاف بنسبة 59,78

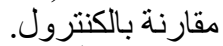

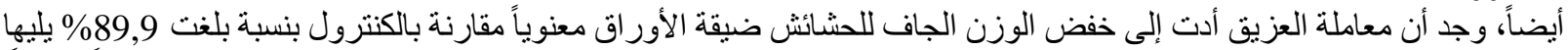

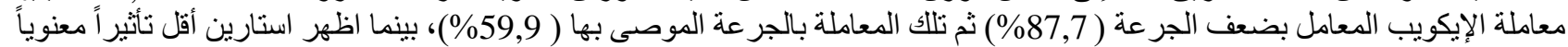

.(\%51)

المجلة العلمية لكلية الزراعة - جامعة القاهرة - المجلا(64) العدد الثالث ( يوليو 2013) :-331-322. 